\title{
Enfermería Brasileña: Herencia de Florence Nightingale en su concepción profesional
}

\section{Brazilian nursing: heritage of the professional conception of Florence Nightingale} \section{Nightingale \\ Maria Angélica de Almeida Peres ${ }^{1}$ \\ Ana Paula Costa Lacerda Brandão ${ }^{2}$ \\ Mercedes de Dios Aguado ${ }^{3}$ \\ Lygia Paim ${ }^{4}$}

Enfermagem brasileira: herança da concepção profissional de Florence

\begin{abstract}
${ }^{1}$ Enfermeira Doutora, Professora Associada do Departamento de Enfermagem Fundamental da Escola de Enfermagem Anna Nery/UFRJ. (Autor Principal) Orcid: http://orcid.org/0000-00026430-3540. Correo electrónico: angelica.ufrj@uol.com.br
\end{abstract}

${ }^{2}$ Historiadora, Mestranda em Enfermagem da Escola de Enfermagem Anna Nery/UFRJ, Bolsista Capes. Orcid: http://orcid.org/0000-0002-6255-3714. Correo electrónico: apclacerda@gmail.com

${ }^{3}$ Centro de Salud de Yepes. Área n ${ }^{\circ} 1$ Toledo. Servicio de Salud de Castilla- La Mancha (SESCAM). Orcid: http://orcid.org/0000-0002-0991-7558. Correo electrónico: mded@sescam.jccm.es

${ }^{4}$ Enfermeira Doutora, Professora Titular Aposentada da Escola de Enfermagem Anna Nery/UFRJ. Orcid: https://orcid.org/0000-0002-6203-8704. Correo electrónico: ipaim9@gmail.com

Correspondencia: Maria Angélica de Almeida. Departamento de Enfermagem Fundamental da Escola de Enfermagem Anna Nery/UFRJ

Correo electrónico de contacto: angelica.ufrj@uol.com.br

Cómo citar esta editorial: Peres, M.A.A., Brandão, A.P.C.L., de Dios Aguado, M., \& Paim, L. (2021). Enfermería Brasileña: Herencia de Florence Nightingale en su concepción profesional. Cultura de los Cuidados (Edición digital), 25 ( $\mathrm{N}^{\circ}$ esp.). Recuperado de http://dx.doi.org/10.14198/cuid.2021.esp.05

Recibido:23/11/2020 Aceptado: 09/02/2021

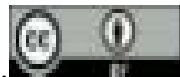

\section{RESUMEN}

Objetivo: Describir la influencia del modelo de cuidado de Florence Nightingale en la formación académica de la enfermería en Brasil y buscar su acercamiento a la cultura brasileña desde el inicio de su 
Revista científica de la Asociación de Historia y Antropología de los Cuidados (Universidad de Alicante)

construcción profesional. Método: En el año 2020, con motivo del bicentenario del nacimiento de Nightingale, surge la narrativa como herramienta para reinterpretar y reflexionar sobre su pensamiento pionero, dentro del desarrollo de la Enfermería Contemporánea Resultados: El texto describe cómo desde principios de la segunda década del siglo $\mathrm{XX}$, el pensamiento de Florence Nightingale influyó en la enseñanza teorico-práctica de la enfermería en Brasil. El modelo educativo implementado por enfermeras estadounidenses fue estandarizado a nivel nacional por el gobierno brasileño, lanzando como base científica el reconocimiento del modelo profesional ingles dentro de la formación de las enfermeras, con características de diseño norteamericanas y con una dirección a culturada. Conclusiones: La narrativa describe el desarrollo de la enfermería contemporánea en Brasil, cuyo modelo se fundamentó en los principios de Nightingale, sin perder las señas de identidad de cultural y siguiendo la influencia de la Escuela de Enfermeras Dona Anna Nery, creada en 1923.

Palabras clave: Historia de la enfermeira; educación en enfermeira; enfermería.

\section{ABSTRACT}

Objective: To describe the trajectory of the nightingalean nursing teaching model in use in Brazil and to seek a possible approach to Brazilian culture in the beginning of this professional construction. Method: Narrativity comes as a tool to reinterpret and reflect on the spread of Contemporaneous Nursing and the importance of the pioneer Florence Nightingale on her bicentenary of birth, celebrated this year of 2020. Results: The text describes how the thinking of Florence Nightingale influenced the teaching and practice of nursing in Brazil, from the beginning of the second decade of the twentieth century, and the teaching model implemented by American nurses with the Brazilian government, nationally standardized, launching as scientific bases the professional recognition of training nurses with the characteristic design, not only of the original English model, since the direction was acculturated with characters from North America. Conclusions: The narrative describes the development of the modern phase of nursing in Brazil with a model idealized in nightingalean principles without losing the characteristic features of the Brazilian culture of the beginning of the 20th century, whose influence was the Dona Anna Nery School of Nurses, created in 1923.

Keywords: History of nursing; nursing education; nursing.

\section{RESUMO}

Objetivo: Descrever em trajetória do modelo de ensino de enfermagem nightingaleano em uso no Brasil e buscar uma aproximação possível com a cultura brasileira nos primórdios dessa construção profissional. Método: A narratividade vem como uma ferramenta para reinterpretar e refletir sobre a difusão da Enfermagem Contemporânea e a importância da pioneira Florence Nightingale, em particular, por ocasião de seu bicentenário de nascimento, celebrado neste ano de 2020. Resultados: O texto vem descrever como o pensamento de Florence Nightingale influenciou o ensino e a prática de enfermagem no Brasil, a partir do início da segunda década do século XX, e o modelo de ensino implantado por enfermeiras norte-americanas junto ao governo brasileiro, padronizado nacionalmente, lançando como bases científicas o reconhecimento profissional de formação de enfermeiras com o desenho característico, não apenas do original modelo inglês, uma vez que a direção estava aculturada de caracteres provenientes da 
América do Norte. Conclusões: A narrativa descreve o desenvolvimento da fase contemporânea da enfermagem no Brasil com um modelo idealizado em princípios nightingaleanos sem perder os traços característicos da cultura brasileira própria do início do século XX, cujo influenciador foi a Escola de Enfermeiras Dona Anna Nery, criada em 1923.

Palavras-chave: História da enfermagem; ensino de enfermagem; enfermagem.

\section{INTRODUCIÓN}

\section{Primeros indicios del pensamiento Nightingale en Brasil}

Las primeras referencias de Florence Nightingale en Brasil se localizan en los periódicos de mediados del siglo XIX, los cuales destacan la importancia de su modelo de cuidado holístico en la Guerra de Crimea (Brandão et al., 2020).

La prensa brasileña entre 1850 y 1910, difunde los múltiples logros alcanzados por Nightingale, tales como la reducción del número de muertes dentro del hospital de campaña; la apertura de una escuela para enfermeras en Londres, la reforma de los hospitales militares bajo su dirección; sus ideas e influencias dentro de la organización de los servicios de salud pública en la India hasta el año de su muerte, las condecoraciones recibidas y aún más, algunos hechos que resaltaban la importancia de su figura femenina en el ámbito de la salud (Brandão et al, 2020; Goodrick \& Reay, 2010).

La enfermería brasileña durante el siglo XIX no fue vista como una profesión académica y ni siquiera contó con legislación propia. Por el contrario, fue considerada una actividad ordenada por médicos y religiosos. La influencia portuguesa durante el período de la colonización y el imperio hizo de la "Irmandad da Misericórdia" la principal institución para atender a los necesitados, siendo los hospitales de Santas Casas diseñados para recibir a los enfermos, en su mayoría personas sin hogar (Peres \& Barreira, 2009).

A partir de 1852 las Hermanas de la Caridad de San Vicente de Paúl fueron traídas de Francia para organizar el servicio de enfermería, convirtiéndose en el principal ejemplo de buena enfermería en el país. Del mismo modo, otras órdenes religiosas se establecieron en el Brasil y asumieron el servicio de enfermería en las instituciones de salud, construyendo un conocimiento jerárquico de enfermería centrado en los principios católicos, la religión oficial del país (Carlos \& Germano, 2011; Padilha, 1999).

Por aquel entonces, el hospital era un lugar inmundo, insalubre e ineficaz para la curación de los enfermos, situación que, durante la segunda mitad del siglo XIX, evolucionó gradualmente gracias a las reformas emprendidas por el emperador Dom Pedro II, las cuales introdujeron mejoras urbanísticas, así como nuevas estrategias sanitarias, con la finalidad de reducir el elevado número de enfermedades infecciosas que asolaban a la población en general y a la que todavía vivía bajo la esclavitud (Padilha, 1998).

Los médicos de la época viajaban a Francia para especializarse y dirigían las instituciones sanitarias, sin embargo, el personal de enfermería realizó tareas subordinadas, ocupando puestos de trabajo en función de los avances de la medicina. Así pues, el personal de enfermería contaba con formación, cualificación, ni actualización, siendo muy común que las personas analfabetas, pobres $\mathrm{y}$ antiguos esclavos desempeñasen las labores de enfermería, eso cuando no habían sido previamente contratados como sirvientes y 
Revista científica de la Asociación de Historia y Antropología de los Cuidados (Universidad de Alicante)

posteriormente desarrollaban labores de enfermería (Mott, 1999; Padilha, 1998).

El final del siglo XIX supuso un período convulso para el pueblo brasileño, debido a las revoluciones que lograron el fin de la esclavitud en 1888 y la Proclamación de la República al año siguiente. En el período republicano, muy influenciado por las ideas positivistas, colocó a la ciencia médica en una posición prominente dentro de la sociedad, hecho que aumentó el interés por la salud pública y abrió espacio para nuevos agentes sanitarios dentro de ese escenario (Mello, 2009).

\section{DESARROLLO DEL TEMA}

\section{Génesis de la enfermería contemporánea en brasil}

Las noticias en los periódicos de 1850 a 1910 en Brasil, destacaban las preocupaciones de Nightingale por el cuidado de los enfermos, así como las desigualdades existentes en materia de salud, las cuales tenían su origen en conflictos político-sociales (Brandão et al., 2020). Puede afirmarse que una de sus principales contribuciones a la naciente profesión enfermera fue identificar el rol del personal de enfermería dentro del cuidado holístico del enfermo diferenciándolo del rol del médico, siendo otra introducir un modelo de formación académica con base científica mediante sus escritos teorizantes (Brandão \& Galluzzi, 2019).

Aunque los informes sobre la vida y las actividades de Florence Nightingale se difundieron por las principales ciudades del Brasil a mediados del siglo XIX, la perspectiva de su modelo enfermero no llegó a la élite brasileña. Sin embargo, la formación académica del personal de enfermería se inició en 1890, cuando se crea la primera escuela de enfermería en el país, aunque el modelo adoptado no fue el difundido por Florence Nightingale (Brandão et al., 2020; Padilha, 1999).
Algunos médicos brasileños se interesaron por la creación de escuelas de enfermería, aunque no existen estudios que revelen cuántas se llevaron a cabo y se desconoce sí la enseñanza enfermera se realizó siguiendo el modelo de Nightingale, con la excepción del Hospital Samaritano de São Paulo, que contrató enfermeras inglesas y abrió un curso para enfermeras alrededor de 1892. Se sabe que hasta 1905 se habían formado un total de 5 enfermeras y, por lo tanto, ejercieron ninguna influencia en la enfermería del país. Luego, la enfermería de esos años mantuvo una ocupación de escaso valor social y su educación académica no era un requisito para desempeñar su labor asistencial (Mott, 1999).

Florence Nightingale creó los fundamentos de la profesión enfermera, estableciendo pautas y estándares para la enseñanza teórico-práctica de la enfermería. $\mathrm{Al}$ observar sus escritos, se descubre que fue la primera investigadora dentro del campo de la enfermería, basando su investigación en la práctica basada en la evidencia, lo que aportó un enfoque organizativo y operativo a los hospitales civiles y militares de la época, inclusive contribuyó al desarrollo de la salud pública, siendo considerada por muchos investigadores como una verdadera administradora de la salud. Florence Nightingale logró elevar el nivel de la profesión y vislumbró que "la educación formalizada es un componente necesario de la preparación de las enfermeras" (Dossey, 2010).

A principios del siglo $\mathrm{XX}$, la salud de la población brasileña seguía siendo precaria y la pandemia de gripe española, que provocó la muerte del presidente de la república, dentro de una estadística del 66\% de la población de la capital del país, la ciudad de Río de Janeiro demostró aún más que los brasileños sufrían las consecuencias del largo período de esclavitud. La reciente liberación de los esclavos trajo a los grandes 
centros un gran número de personas en busca de empleo y vivienda. La población creció y el gobierno trató de acompañar sólo el desarrollo económico y social, cada vez más dependiente de trabajadores sanos (Goulart, 2005).

Entre las actividades del gobierno federal para el desarrollo del país, la salud pública recibió una atención especial, de manera que la implantación del modelo de la enfermera de salud pública desarrollado en norteamericana empezó a considerarse como una posible solución para la educación sanitaria de la población (Sauthier y Barreira, 1999).

\section{Escuelas de enfermería y fundación rockefeller en Brasil}

La alianza entre el Brasil y los Estados Unidos favoreció la llegada de una misión de enfermeras para actuar dentro de la reforma de la salud, se creó una escuela de enfermeras y se comenzó un proyecto para desarrollar la Enfermería Contemporánea en el país, proyecto muy similar a otros ya patrocinados por la Fundación Rockefeller en América Central y del Sur (Peters et al., 2020). Esta Fundación invirtió mucho en países en los que era necesaria una inyección de capital y, en el caso de Brasil en particular, la riqueza natural fue la contrapartida de esta inversión, incluso en el ámbito de la salud pública (Barreira et al., 2001)

La llegada de una misión médica de la Fundación Rockefeller al Brasil en 1916 inició la internalización de la atención de la salud pública y en 1920 se creó el Departamento Nacional de Salud Pública (DNSP) para establecer una reforma (Peters et al., 2020).

En 1923, conforme a los estándares angloamericanos para la educación en enfermería se inauguró la Escuela de Enfermería del DNSP, la cual mantuvo un gran parecido a las escuelas de Estados Unidos, tales como la Escuela de Enfermería del Hospital General de Filadelfia y la Escuela de Enfermería de Vanderblit.

La Fundación Rockefeller, por medio de sus actividades alrededor del mundo, ejerció una gran influencia en la promoción de escuelas de enfermería, siendo el modelo de enseñanza enfermero que difundió fue el creado por Nightingale (Peres \& Padilha, 2014). Dicho modelo fue el adoptado por la Escuela de Enfermería de DNSP, más tarde llamada Escuela de Enfermería Anna Nery de la Universidad de Brasil. Sin embargo, antes de que la escuela de enfermería se inaugurará en 1923, la Enfermería Contemporánea inició su andadura, por medio del servicio de enfermería de salud pública, creado en 1922, el cual contó con enfermeras norteamericanas cuyo liderazgo fue reconocido por el gobierno brasileño (Barreira et al., 2001).

La escuela poseía un plan de estudios ajustado al Plan de Estudios Estándar de Enfermería, pero diseñado a su vez para atraer candidatas inteligentes $\mathrm{y}$ competentes, ya que la formación era exclusiva para mujeres en régimen de internado, a tiempo completo. Para elevar el estatus de todas las escuelas de enfermería se llevaba a cabo una enseñanza teóricopráctica y la dirección estuvo a cargo de una enfermera (Gray, 1918). Durante el curso, en la escuela, existían unos rituales implantados tales como la ceremonia de graduación, la ceremonia de recepción del birrete y el juramento solemne, estos fueron los mismos que los creados por Nightingale en la escuela de Londres, los cuales a su vez fueron llevados a cabo dentro de las escuelas norteamericanas (Peters et al., 2020).

Los documentos de la escuela revelan una fuerte identidad con la figura de Florence Nightingale, pues su imagen quedo inmortalizada en una estatua de cuerpo entero, donde se la representa sosteniendo 
una lámpara. Dicha estatua fue colocada en el local del internado (residencia) para las enfermeras siempre tuvieran presente su imagen como principal referente a seguir. Además, la lámpara, símbolo de la enfermería contemporánea, la cual hace referencia a Florence Nightingale, forma parte de la simbología de la bandera de la escuela, ya que a partir de 1926 pasó a denominarse Escuela de Enfermeras Dona Anna Nery, enfermera voluntaria brasileña de la Guerra del Paraguay (Dias et al., 2016).

Otro elemento de identidad profesional que rememora la escuela de enfermeras creada por Nightingale es el uniforme que llevaban las alumnas $\mathrm{y}$ profesores de la escuela. El vestido a rayas azules y blancas con el delantal y la gorra blanca de las estudiantes, así como el vestido blanco de los profesores de enfermería eran similares al patrón de enseñanza difundido en todo el mundo, especialmente con la ayuda de la Fundación Rockefeller (Peres \& Padilha, 2014; Peters et al., 2020).

En los primeros años de funcionamiento de la escuela, las enfermeras fueron muy solicitadas y absorbidas para llevar a cabo la reforma sanitaria. Dentro de las actividades de salud pública, se puso énfasis en la educación sanitaria, en las actividades destinadas a la prevención de enfermedades y en la supervisión de otros trabajadores de enfermería dentro de los servicios federales. Poco a poco, este nuevo modelo de enfermera se introdujo en los hospitales, transformando su modelo organizativo con relación a la enfermería dentro del equipo de salud (Santos y Barreira, 2008).

La salida de las enfermeras brasileñas a los cursos de postgrado en los Estados Unidos fue una estrategia gubernamental para lograr formar un grupo de líderes de enfermería, capaces de dirigir la transformación que suponía poner en práctica la reforma de la salud, la cual debía extenderse a todo el conjunto del sistema de salud. Sin embargo, el hecho de ser una profesión exclusivamente femenina fue un impedimento, ya que se tuvieron que enfrentare a los límites impuestos por la sociedad brasileña de la época.

Así, la inserción de enfermeras como profesoras y directoras de escuelas de enfermería mostró que la enseñanza de Nightingale debía recaer en manos de las enfermeras para formar a nuevos profesionales. Luego, el comportamiento de estas mujeres se caracterizó por un respeto a la jerarquía, unos valores morales rígidos y el mantenimiento de una uniformidad exquisita en sus vestimentas y cabello, características que se ajustaban a los estándares sociales de las clases más altas e influyentes (Santos \& Barreira, 2008).

Las mujeres brasileñas, gradualmente se interesaron por la profesión enfermera, hecho que motivó un importante reconocimiento social en la década de 1920. A su vez, la creación de una Asociación de Enfermeras Brasileñas en 1926 permitió el acceso de la enfermería brasileña al Consejo Internacional de Enfermeras (CIE) y motivó una corriente de profesionalización femenina por América del Sur (Carvalho, 2002).

Durante los nueve años (1922-1931) que la misión norteamericana de enfermeras dirigida por la enfermera Ethel Parsons permaneció en el Brasil, colocó a las nuevas profesionales en una posición diferente a la de las religiosas y otras enfermeras del país. La Escuela de Enfermería Dona Anna Nery fue preparada y designada para formar parte intrínseca de la universidad en 1931. Durante el período de difusión de la Enfermería Contemporánea, la Fundación Rockefeller mantuvo su apoyo político y financiero tanto en la Escuela Dona Anna Nery como a otras escuelas de enfermería, de tal modo que la Fundación Rockefeller también ofreció becas para que las 
enfermeras asistiesen a cursos de posgrado en instituciones estadounidenses (Korndörfer, 2019).

La enfermería Contemporánea en el Brasil ha alcanzado un nivel diferenciado y respetado por los profesionales médicos y los líderes de los servicios de salud. A pesar de las dificultades a las que se enfrentaba la naciente profesión femenina debido a las limitaciones de la mujer en la sociedad brasileña, sus pioneros asumieron el compromiso de su desarrollo y siguieron las enseñanzas que impregnan los escritos dejados por Florence Nightingale, que hasta hoy constituyen el diferencial más interiorizado de liderazgo que aún hoy ejerce la Escuela de Enfermería Anna Nery.

\section{CONCLUSIONES}

El modelo de cuidado de enfermería promovido por Nightingale se conformó en base a sus convicciones con relación al trabajo que debía desempeñar la enfermera dentro del cuidado holístico de la persona y la influencia que el entorno ejercía dentro de su proceso de curación. Estos valores acreditaron la labor de enfermería, la cual afianzó su conocimiento y cualificación gracias a la formación académica de calidad que Nightingale promovió. Sus pensamientos y enseñanzas fueron una distinción notable, para el modelo educativo de las mujeres en el siglo XIX.

Esta narración ha intentado describir el legado que Brasil recibio con relación al modelo de cuidado defendido por Nightingale. A su vez, describe la inversión pública que fue necesario implementar para lograr una formación académica de la profesión enfermera durante la segunda década del siglo XX. Así el modelo angloamericano de enfermería fue el primero en ser reconocido nacionalmente $y$, por fuerza de ley, se hizo oficial en el país.
La Enfermería Contemporánea estuvo presente en la Escuela de Enfermería Anna Nery, además tomó los caracteres de los estudios diferenciados para enfermeros, en el momento en que avanzaba como un anexo de la Universidad de Brasil, siendo admitida en los años 1937. La singularidad del pensamiento de Nightingale nunca estuvo alejado de la formación académica que promueve la Escuela

Desde sus inicios la Escuela de Enfermería Anna Nery adopto el pensamiento de Nightingale e hizo suyos los valores de RESPETO y REVERENCIA, proponiendo prácticas ligadas al modelo de cuidado holístico defendido por Nightingale, el cual lo considera como una brújula que marca el camino que debe seguir el personal de enfermería en el desarrollo de su labor asistencial.

La vida de Florence Nightingale es objeto de estudio entre los contenidos de los programas de enseñanza de Historia de la Enfermería y su trayectoria profesional se ejemplifica en formas de lecturas de comportamiento. Se trata de una herencia inmaterial e infinita, que sólo depende del vigor de la enfermería brasileña, la cual nunca ha estado alejada del pensamiento de Nightingale e inclusive es considerada una marca de identidad entre las enfermeras y los enfermeros de la sociedad brasileña.

\section{BIBLIOGRAFÍA}

Barreira, I. de A., Sauthier, J., \& Baptista, S. de S. (2001). O movimento associativo das enfermeiras diplomadas brasileiras na $1^{\mathrm{a}}$ metade do século 20. Revista Brasileira de Enfermagem, 54(2), 157-173. https://doi.org/10.1590/S003471672001000200002

Brandão, A. P. da C. L., \& Galluzzi, M. L. (2019). Aspectos epistemo-cognitivofilosóficos no ideário de Florence 
Revista científica de la Asociación de Historia y Antropología de los Cuidados (Universidad de Alicante)

Nightingale (1820-1910). Revista

Scientiarum Historia, 2, 1-10.

Brandão, A. P. da C. L., Peres, M. A. de A., Aperibense, P. G. G. de S., Lopes, R. O. P., Santos, J. de C., \& Brandão, M. A. G. (2020). Evidence of nursing patterns of knowing communicated by the brazilian press before Florence Nightingale's model. Revista Brasileira de Enfermagem, 73(suppl 5). https://doi.org/10.1590/0034-7167-20190790

Carlos, D. J. D., \& Germano, R. M. (2011). Enfermagem: história e memórias da construção de uma profissão. Revista Mineira de Enfermagem, 15, 513-521.

Carvalho, A. C. de. (2002). Associação Brasileira de Enfermagem-1926/1976: documentário. Revista Brasileira de Enfermagem, 55(3), 249-263. https://doi.org/10.1590/S0034-

71672002000300003

Dias, N. L., Carvalho, M. da S., Paim, L., AperibenseI, P. G. G. de S., \& Peres, M. A. de A. (2016). Monumentos e personagens históricos: preservação da identidade profissional da enfermagem em espaço acadêmico. HERE, 7(2), 423-439.

Dossey, B. M. (2010). Florence Nightingale. Journal of Holistic Nursing, 28(1), 10-35. https://doi.org/10.1177/0898010109356474

Goodrick, E., \& Reay, T. (2010). Florence Nightingale Endures: Legitimizing a New Professional Role Identity. Journal of Management Studies, 47(1), 55-84. https://doi.org/10.1111/j.14676486.2009.00860.x

Goulart, A. da C. (2005). Revisitando a espanhola: a gripe pandêmica de 1918 no Rio de Janeiro. História, Ciências, SaúdeManguinhos, 12(1), 101-142. https://doi.org/10.1590/S010459702005000100006

Gray, C. E. (1918). The Standard Curriculum for Schools of Nursing. The
American Journal of Nursing, 18, 790-794. https://www.jstor.org/stable/pdf/3405660.p df

Korndörfer, A. P. (2019). A Fundação Rockefeller e a formação de quadros para a enfermagem (Brasil: 1917-1951). Nuevo Mundo Mundos Nuevos. https://doi.org/10.4000/nuevomundo.76226

Mello, M. T. C. De. (2009). A Modernidade Republicana *. Tempo, 13, 15-31. http://www.scielo.br/pdf/tem/v13n26/a02v1 326.pdf

Mott, M. L. (1999). Revendo a História da Enfermagem em São Paulo (1890- 1920). Cadernos Pagu, 327-355.

Padilha, M. I. C. de S. (1998). A mística do silêncio: a prática de enfermagem na Santa Casa de Misericórdia do Rio de Janeiro no $\begin{array}{llll}\text { século XIX (1st ed.). } & \text { Ed. }\end{array}$ Universitária/UFPel.

Padilha, M. I. C. de S. (1999). As representações da história da enfermagem na prática cotidiana atual. Revista Brasileira de Enfermagem, 52(3), 443-454. https://doi.org/10.1590/S003471671999000300014

Peres, M. A. de A., \& Barreira, I. de A. (2009). Desenvolvimento da assistência médica e de enfermagem aos doentes mentais no Brasil: os discursos fundadores do hospício. Texto \& Contexto Enfermagem, 18(4), 635-642. https://doi.org/10.1590/S010407072009000400004

Peres, M. A. de A., \& Padilha, M. I. C. de S. (2014). Uniform as a sign of a new nursing identity in Brazil (1923-1931). Escola Anna Nery - Revista de Enfermagem, 18(1). https://doi.org/10.5935/14148145.20140017

Peters, A. A., Peres, M. A. de A., \& D’Antonio, P. (2020). Influências do Sistema Anglo-Americano de Ensino no Brasil: Contribuições da Missão Parsons 
(1921-1925). OJIN: The Online Journal of Issues in Nursing, 25, 3-11.

Santos, T. C. F., \& Barreira, I. de A. (2008). A mulher e a enfermeira na nova ordem social do Estado Novo. Texto \& Contexto Enfermagem, 17(3), 587-593. https://doi.org/10.1590/S0104-

07072008000300021

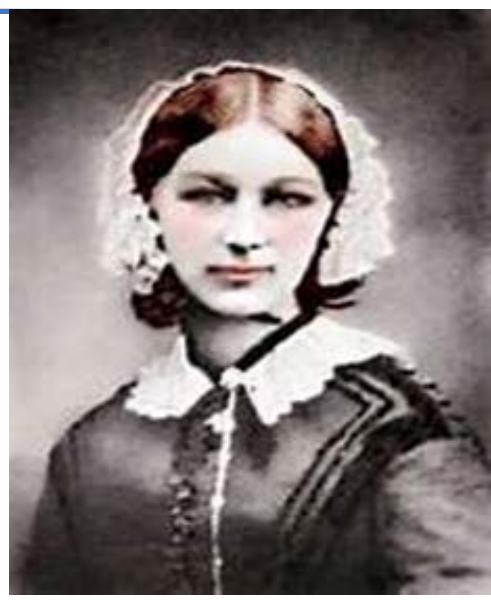

